\title{
Harmonic generation at a nonlinear imperfect joint of plates by the SO Lamb wave incidence
}

$\operatorname{AUTHOR}(S)$ :

Mori, Naoki; Biwa, Shiro; Kusaka, Takayuki

\section{CITATION:}

Mori, Naoki ... [et al]. Harmonic generation at a nonlinear imperfect joint of plates by the So Lamb wave incidence. Journal of Applied Mechanics 2019, 86(12): 121003.

ISSUE DATE:

2019-12

URL:

http://hdl.handle.net/2433/254631

\section{RIGHT:}

Copyright (c) 2019 by ASME. This manuscript version is made available under the CC-BY-NC-ND 4.0 license

http://creativecommons.org/licenses/by-nc-nd/4.0/; The full-text file will be made open to the public on 17 September 2020 in accordance with publisher's 'Terms and Conditions for Self-Archiving'., この論文は出版社版でありません。引 用の際には出版社版をご確認ご利用ください。; This is not the published version. Please cite only the published version. 


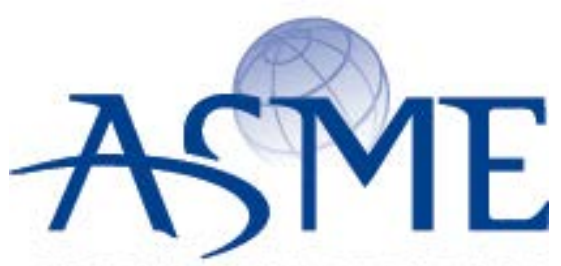

\section{American Society of Mechanical Engineers}

SETTING THE STANDARD

\section{ASME Accepted Manuscript Repository}

\section{Institutional Repository Cover Sheet}

ASME Paper Title: Harmonic Generation at a Nonlinear Imperfect Joint of Plates by the SO Lamb Wave Incidence

Authors: $\quad$ Naoki Mori, Shiro Biwa, Takayuki Kusaka

ASME Journal Title: Journal of Applied Mechanics

Volume/Issue Volume $86 /$ Issue 12

Date of Publication (VOR* Online) September 17, 2019

ASME Digital Collection URL: https://asmedigitalcollection.asme.org/appliedmechanics/article-abstract/86/12/121003/958391/

DOI: $\quad$ https://doi.org/10.1115/1.4044457

*VOR (version of record) 
Journal of Applied Mechanics

\section{Harmonic Generation at a Nonlinear Imperfect Joint of Plates by the So Lamb Wave Incidence}

\section{Naoki Mori ${ }^{1}$}

Department of Mechanical Engineering, Ritsumeikan University, 1-1-1 Noji-higashi, Kusatsu, Shiga 525-8577, Japan

e-mail: nmori@fc.ritsumei.ac.jp

\section{Shiro Biwa}

Department of Aeronautics and Astronautics, Kyoto University, Katsura, Nishikyo-ku, Kyoto 615-8540, Japan e-mail: biwa@kuaero.kyoto-u.ac.jp

\section{Takayuki Kusaka}

Department of Mechanical Engineering, Ritsumeikan University, 1-1-1 Noji-higashi, Kusatsu, Shiga 525-8577, Japan

e-mail: kusaka@se.ritsumei.ac.jp

\footnotetext{
${ }^{1}$ Corresponding author.
} 


\begin{abstract}
Nonlinear interaction of Lamb waves with an imperfect joint of plates for the incidence of the lowest-order symmetric (SO) Lamb wave is investigated by perturbation analysis and time-domain numerical simulation. The imperfect joint is modeled as a nonlinear spring-type interface, which expresses interfacial stresses as functions of the displacement discontinuities. In the perturbation analysis, under the assumption of weak nonlinearity, the second-harmonic generation at the joint is examined in the frequency domain by the thin-plate approximation using extensional waves. As a result, the amplitude of the second-harmonic extensional wave is shown to be in good agreement with the result of the SO mode in a low frequency range. However, it is found that the thin-plate approximation does not reproduce the amplification of the second-harmonic SO mode, which occurs due to the resonance of the joint. Furthermore, the time-domain analysis is performed by the elastodynamic finite integration technique (EFIT). When the amplitude of the incident wave is relatively large, the fundamental wave and the second harmonic exhibit different behavior from the results by the perturbation analysis. Specifically, if the incident amplitude is increased, the peak frequency of the second-harmonic amplitude becomes low. The transient behavior of the nonlinear interaction is also examined and discussed based on the results for the weak nonlinearity.
\end{abstract}

Keywords: Nonlinear Lamb wave; Imperfect joint; Harmonic generation; Resonance

JAM-19-1334 Mori et al. 


\section{Introduction}

When a structural component containing a crack-type defect is subjected to compressive loading or temperature variation, the crack surfaces often get in partial contact with each other. Elastic waves interact with such a contacting interface in a complicated manner. The reflection and transmission behavior of elastic waves at the contacting interface depends on the applied static pressure as well as the condition of the contacting surfaces [1-4]. For the normal incidence of a bulk wave, for example, the reflection coefficient at a contacting interface between two blocks decreases monotonically with increasing contact pressure. This feature is theoretically explained by the increase of the linear interfacial stiffness with increasing contact pressure. The decrease of the reflection coefficient is often troublesome in the nondestructive evaluation of partially closed cracks by the ultrasonic pulse-echo method.

Elastic waves exhibit nonlinear interaction with an imperfect interface when their amplitudes are large. It was shown in previous studies that when an elastic wave of relatively high amplitude interacts with a contacting interface between solids, nonlinear frequency components such as higher harmonics and sub-harmonics are generated at the interface due to the contact acoustic nonlinearity (CAN) [5-14]. The nonlinear behavior of elastic waves was reported for not only contacting interfaces but also for adhesive interfaces [15-17] and multilayered structures [18, 19].

Lamb waves are known as elastic guided waves propagating in plates and their propagation distance is relatively long compared to bulk waves. The effects of material nonlinearity on the Lamb wave propagation were investigated extensively in previous studies [20-24]. Lamb waves show cumulative harmonic generation on specific conditions depending on their dispersion and multi-modal properties. Furthermore, the CAN leads to harmonic generation at a partially closed defect when subjected to the Lamb wave incidence [25-33]. Hong et al. [27] measured the signals of the second-harmonic Lamb waves generated at a fatigue crack, estimating the crack location using their temporal information. More recently, Yang et al. [33] have measured the second harmonic generated at fatigue cracks for the incidence of the low-frequency Lamb wave, reasonably reproducing its directivity pattern by the finite element simulation. The nonlinear behavior of the Lamb wave at a closed crack has been numerically simulated by Shkerdin and Glorieux [25], Shen and Giuriutiu [26], and many other papers [29-33], based on the modeling of clapping contacts.

In order to explain the nonlinear behavior of elastic waves, various models were proposed for contacting rough surfaces in previous studies. The interfacial stress components are usually expressed as functions of the gap distance between the nominal 
surfaces and the tangential displacement. A typical model for an imperfect interface is a piecewise linear spring-type model $[14,16]$, in which the stiffness of the interface takes different values in tension and compression states, for example. Another manner to model contacting surfaces is the penalty method, which is used in numerical simulations such as a finite element simulation [26, 30, 31]. However, these models do not reproduce the nonlinear pressure-gap relation of contacting rough surfaces, which is necessary to show the pressure dependence of the reflection and transmission characteristics of elastic waves. The nonlinear interface model $[34,35]$ can take into account this effect. However, fundamental aspects of the Lamb wave interaction with a nonlinear interface has not been sufficiently revealed yet.

The objective of this study is to investigate the harmonic generation behavior of Lamb waves at a nonlinear joint of plates. To this purpose, the imperfect joint is modeled as a nonlinear spring-type interface, which expresses interfacial stresses as functions of the displacement gaps [34, 35]. The interaction of Lamb waves with a linear spring-type interface has been reported in the previous papers [36-39]. Recently, the second-harmonic generation behavior for the incidence of the lowest-order symmetric (S0) Lamb wave has been shown by the perturbation analysis in the frequency domain using the hybrid finite element method (HFEM) [40]. In the present study, the second-harmonic generation at the imperfect joint is examined based on the thin-plate approximation using extensional waves. The perturbation analysis is carried out under the assumption of weak nonlinearity, and the obtained second-harmonic amplitude is compared to the perturbation analysis results of the S0 mode. Furthermore, a time-domain numerical simulation is performed by the elastodynamic finite integration technique (EFIT) [41], in which the assumption of the weak nonlinearity is not required. The nonlinear interaction at relatively high incident amplitude is particularly explored.

The outline of this paper is as follows. In Section 2, the problem considered in this study is described. In Section 3, the formulation of the perturbation analysis for the S0 Lamb wave is given, and the approximated formulation is explained based on the thin-plate theory of extensional waves. In Section 4, the details of the modeling for the time-domain analysis are presented. In Section 5, the result of the perturbation analysis for the second-harmonic extensional wave is shown and used to discuss the perturbation analysis results for the S0 mode. Furthermore, the results of the time-domain analysis for sinusoidal wave incidence are shown. The effect of the incident amplitude on the transmitted fundamental wave and second harmonic is investigated. The transient behavior of the second-harmonic generation for pulse wave incidence is also shown. 


\section{Statement of the Problem}

As shown in Fig. 1, two semi-infinite, homogeneous and isotropic linear elastic plates of thickness $d$ occupy the regions $x_{1}<0,\left|x_{2}\right|<d / 2$ and $x_{1}>0,\left|x_{2}\right|<d / 2$, respectively, in the Cartesian $x_{1}-x_{2}-x_{3}$ coordinate system. The plates are under the plane-strain condition in the $x_{1}-x_{2}$ plane and are imperfectly jointed at $x_{1}=0$. The propagation behavior of Lamb waves in the plates obeys the two-dimensional Navier equation

$$
\left(c_{\mathrm{L}}^{2}-c_{\mathrm{T}}^{2}\right) \frac{\partial}{\partial x_{\alpha}}\left(\frac{\partial u_{\gamma}}{\partial x_{\gamma}}\right)+c_{\mathrm{T}}^{2} \frac{\partial}{\partial x_{\gamma}}\left(\frac{\partial u_{\alpha}}{\partial x_{\gamma}}\right)=\frac{\partial^{2} u_{\alpha}}{\partial t^{2}},
$$

where $t$ denotes time, $c_{\mathrm{L}}$ and $c_{\mathrm{T}}$ the velocities of longitudinal and transverse waves, respectively, and $u_{\alpha}\left(x_{1}, x_{2}, t\right)$ displacement components $(\alpha=1,2)$. The two-dimensional summation convention is used in Eq. (1). On the plate surfaces $\left|x_{2}\right|=d / 2$, the traction-free boundary condition, i.e. $\sigma_{\alpha 2}=0$, is applied, where $\sigma_{\alpha \gamma}\left(x_{1}, x_{2}, t\right)$ is stress components obtained from Hooke's law $(\alpha, \gamma=1,2)$.

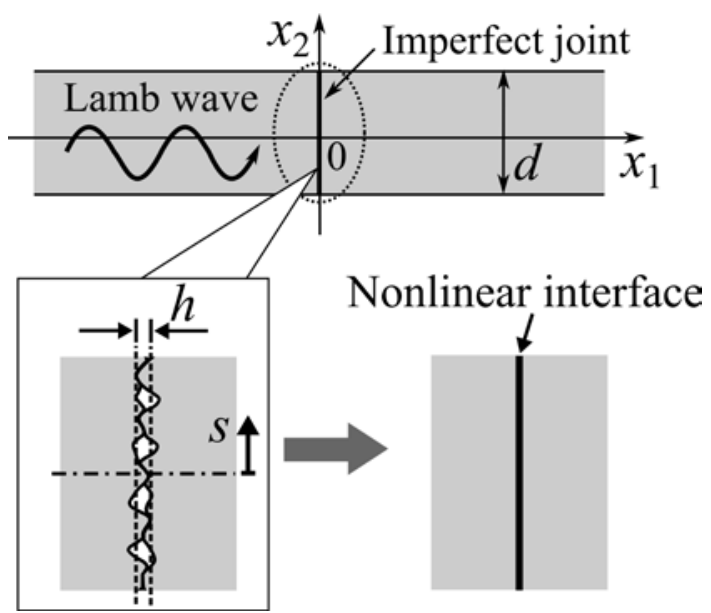

Fig. 1 Nonlinear imperfect joint of isotropic linear elastic plates at $x_{1}=0$

A nonlinear interface model is used to model an imperfect joint $x_{1}=0$ [34, 35]. The details of the modeling are described in [35]. The gap distance between the nominal joint surfaces is denoted as $h$, which is assumed to be sufficiently small compared to the wavelength of the incident wave. When the Lamb wave interacts with the interface, the gap distance $h$ varies with time and the tangential displacement $s$ is induced at the interface. The effects of hysteresis and friction are neglected, and the interfacial stress components are given by

$$
\begin{aligned}
& \sigma_{11}\left(0^{ \pm}, x_{2}, t\right)=g_{\mathrm{N}}(h, s), \\
& \sigma_{12}\left(0^{ \pm}, x_{2}, t\right)=g_{\mathrm{T}}(h, s),
\end{aligned}
$$


where $x_{1}=0^{+}\left(x_{1}=0^{-}\right)$represents the right (left) side of the interface, and $g_{\mathrm{N}}$ and $g_{\mathrm{T}}$ are functions of $h$ and $s$. At an equilibrium state, the gap distance and the tangential displacement are expressed as $h=h_{0}$ and $s=0$, respectively. In this study, the normal and tangential stress components in Eqs. (2) and (3) are assumed to be even and odd functions with respect to the tangential displacement $s$, respectively [35].

If the perturbations of $h$ and $s$ from the equilibrium state are sufficiently small, the interfacial stress components of Eqs. (2) and (3) are expressed by the second-order Taylor expansion around the equilibrium state

$$
\begin{aligned}
& \sigma_{11}\left(0^{ \pm}, x_{2}, t\right)=K_{\mathrm{N}}\left(h-h_{0}\right)-K_{\mathrm{NN}}\left(h-h_{0}\right)^{2}-K_{\mathrm{TT}} s^{2}, \\
& \sigma_{12}\left(0^{ \pm}, x_{2}, t\right)=K_{\mathrm{T}} s-K_{\mathrm{NT}}\left(h-h_{0}\right) s,
\end{aligned}
$$

where the coefficients in Eqs. (4) and (5) are the derivatives of the stress components at the equilibrium state

$$
\begin{aligned}
& K_{\mathrm{N}}=\frac{\partial g_{\mathrm{N}}}{\partial h}\left(h_{0}, 0\right), K_{\mathrm{NN}}=-\frac{1}{2} \frac{\partial^{2} g_{\mathrm{N}}}{\partial h^{2}}\left(h_{0}, 0\right), K_{\mathrm{TT}}=-\frac{1}{2} \frac{\partial^{2} g_{\mathrm{N}}}{\partial s^{2}}\left(h_{0}, 0\right), \\
& K_{\mathrm{T}}=\frac{\partial g_{\mathrm{T}}}{\partial s}\left(h_{0}, 0\right), K_{\mathrm{NT}}=-\frac{\partial^{2} g_{\mathrm{T}}}{\partial h \partial s}\left(h_{0}, 0\right),
\end{aligned}
$$

and $K_{\mathrm{N}}$ and $K_{\mathrm{T}}$ are normal and tangential stiffnesses, respectively. The modeling of an imperfect interface which is similar to Eqs. (4) and (5) is shown in [42].

The imperfect joint is subjected to the incidence of the S0 Lamb wave propagating in the $x_{1}$ direction. The incident frequency $f_{0}$ is limited in a low frequency range below the half of the S1 mode cut-off frequency $f_{\mathrm{S} 1}$, i.e.

$$
f_{0}<\frac{f_{\mathrm{S} 1}}{2}=\frac{c_{\mathrm{T}}}{2 d},
$$

in which only the S0 and lowest-order antisymmetric (A0) modes can propagate in the plates. The frequency of the second harmonic $2 f_{0}$ does not exceed the cut-off frequency of the S1 mode. Since the deformation of the S0 mode is dominant in the in-plane $\left(x_{1}\right)$ direction compared to the out-of-plane $\left(x_{2}\right)$ direction, the terms of $\left(h-h_{0}\right) s$ and $s^{2}$ in Eqs. (4) and (5) are assumed to be negligible in this study. Namely, the interfacial stresses in Eqs. (4) and (5) are rewritten as

$$
\begin{aligned}
& \sigma_{11}\left(0^{ \pm}, x_{2}, t\right)=K_{\mathrm{N}}\left(1-\beta\left[u_{1}\right]\right)\left[u_{1}\right], \\
& \sigma_{12}\left(0^{ \pm}, x_{2}, t\right)=K_{\mathrm{T}}\left[u_{2}\right],
\end{aligned}
$$

where $\left[u_{1}\right]=h-h_{0}=u_{1}\left(0^{+}, x_{2}, t\right)-u_{1}\left(0^{-}, x_{2}, t\right),\left[u_{2}\right]=s=u_{2}\left(0^{+}, x_{2}, t\right)-u_{2}\left(0^{-}, x_{2}, t\right)$, and $\beta=$ $K_{\mathrm{NN}} / K_{\mathrm{N}}$. In the present study, the interfacial parameters $K_{\mathrm{N}}, K_{\mathrm{T}}$, and $\beta$ are set to be uniform in the $x_{2}$ direction. Since mode conversion from symmetric to antisymmetric Lamb modes does not occur due to the symmetry of the joint, only the S0 mode can propagate as the second harmonic in the case of the S0 mode incidence.

The material properties of aluminum alloy $\left(c_{\mathrm{L}}=6.4 \mathrm{~km} / \mathrm{s}, c_{\mathrm{T}}=3.17 \mathrm{~km} / \mathrm{s}\right.$, and the JAM-19-1334 Mori et al. 
mass density $\rho=2.7 \times 10^{3} \mathrm{~kg} / \mathrm{m}^{3}$ ) are used in the numerical analysis. The thickness of the plates is set as $d=1 \mathrm{~mm}$. To describe the numerical results in a simple manner, the ratio of the tangential to the normal stiffnesses is fixed as $K_{\mathrm{T}} / K_{\mathrm{N}}=0.3$. This value is within the possible range of the interfacial stiffness ratio (between 0.2 and 0.6 ) which was reported in previous studies [2, 39, 43-45].

\section{Perturbation Analysis}

\subsection{Formulation}

The partial differential equation of Eq. (1) is numerically solved under the nonlinear boundary condition of Eqs. (8) and (9) by the perturbation analysis in the frequency domain [40]. The displacement and stress components are written as complex values, i.e. $u_{\alpha}\left(x_{1}, x_{2}, t\right)=U_{\alpha}\left(x_{1}, x_{2}\right) \exp (-\mathrm{i} \omega t)$ and $\sigma_{\alpha \gamma}\left(x_{1}, x_{2}, t\right)=\Sigma_{\alpha \gamma}\left(x_{1}, x_{2}\right) \exp (-\mathrm{i} \omega t)$, respectively, where $\mathrm{i}=\sqrt{-1}$ and $\omega$ is angular frequency. When a monochromatic wave of angular frequency $\omega_{0}=2 \pi f_{0}$ interacts with a nonlinear interface, higher-order harmonics of angular frequency $n \omega_{0}(n=2,3, \ldots)$ and direct current (DC) component are expected to be generated due to the nonlinear effect. In the perturbation analysis, a nonlinearity parameter $\varepsilon=\beta A_{0}$ is assumed to be sufficiently small, i.e. $\varepsilon<<1$, where $A_{0}$ is the in-plane displacement amplitude of the incident wave on the middle plane of the plate.

The governing equations and the boundary conditions of Eqs. (1), (8), and (9) for fundamental waves at $\omega=\omega_{0}$ are expressed as

$$
\begin{aligned}
& \left(c_{\mathrm{L}}^{2}-c_{\mathrm{T}}^{2}\right) \frac{\partial}{\partial x_{\alpha}}\left(\frac{\partial U_{\gamma}^{\mathrm{F}}}{\partial x_{\gamma}}\right)+c_{\mathrm{T}}^{2} \frac{\partial}{\partial x_{\gamma}}\left(\frac{\partial U_{\alpha}^{\mathrm{F}}}{\partial x_{\gamma}}\right)=\left(-\mathrm{i} \omega_{0}\right)^{2} U_{\alpha}^{\mathrm{F}}, \\
& \Sigma_{11}^{\mathrm{F}}\left(0^{ \pm}, x_{2}\right)=K_{\mathrm{N}}\left[U_{1}^{\mathrm{F}}\right], \\
& \Sigma_{12}^{\mathrm{F}}\left(0^{ \pm}, x_{2}\right)=K_{\mathrm{T}}\left[U_{2}^{\mathrm{F}}\right],
\end{aligned}
$$

respectively, where the superscript $\mathrm{F}$ represents the components of the fundamental waves. The reflection and transmission for the S0 mode incidence at $\omega=\omega_{0}$ are analyzed by Eqs. (10)-(12), and the interfacial displacement gap by the fundamental wave $\left[U_{1}{ }^{\mathrm{F}}\right]$ is obtained as a complex value $\left[U_{1}{ }^{\mathrm{F}}\right]=Y_{\mathrm{N}}=Y_{1} \exp \left(-\mathrm{i} \phi_{1}\right)$, where $Y_{1}$ and $\phi_{1}$ are real numbers. Under the assumption of weak nonlinearity, the quadratic term $\left[u_{1}\right]^{2}$ in Eq. (8) is replaced by $\left[\operatorname{Re}\left\{Y_{\mathrm{N}} \exp \left(-\mathrm{i} \omega_{0} t\right)\right\}\right]^{2}$, and Eq. (8) is rewritten as

$$
\sigma_{11}\left(0^{ \pm}, x_{2}, t\right)=K_{\mathrm{N}}\left[u_{1}\right]-\beta K_{\mathrm{N}}\left[\operatorname{Re}\left\{Y_{\mathrm{N}} \exp \left(-\mathrm{i} \omega_{0} t\right)\right\}\right]^{2} .
$$

The second term in the right-hand side of Eq. (13) serves as the source of the second harmonics and the DC components. In the frequency domain, the governing equations and the boundary conditions for the second harmonics at $\omega=2 \omega_{0}$ are expressed as

$$
\left(c_{\mathrm{L}}^{2}-c_{\mathrm{T}}^{2}\right) \frac{\partial}{\partial x_{\alpha}}\left(\frac{\partial U_{\gamma}^{\mathrm{S}}}{\partial x_{\gamma}}\right)+c_{\mathrm{T}}^{2} \frac{\partial}{\partial x_{\gamma}}\left(\frac{\partial U_{\alpha}^{\mathrm{S}}}{\partial x_{\gamma}}\right)=\left(-\mathrm{i} 2 \omega_{0}\right)^{2} U_{\alpha}^{\mathrm{S}},
$$




$$
\begin{aligned}
& \Sigma_{11}^{\mathrm{S}}\left(0^{ \pm}, x_{2}\right)=K_{\mathrm{N}}\left[U_{1}^{\mathrm{S}}\right]-\frac{\beta K_{\mathrm{N}} Y_{1}^{2}}{2} \exp \left\{-\mathrm{i}\left(2 \phi_{1}\right)\right\}, \\
& \Sigma_{12}^{\mathrm{S}}\left(0^{ \pm}, x_{2}\right)=K_{\mathrm{T}}\left[U_{2}^{\mathrm{S}}\right],
\end{aligned}
$$

respectively, where the superscript $S$ represents the second-harmonic components. The above partial differential equations are numerically solved using the hybrid finite element method (HFEM) [36]. The calculation parameters given in [40] are used in the present study.

\subsection{Thin-Plate Approximation}

It was shown in [36] that the reflection and transmission characteristics of the S0 mode at the imperfect joint in a low frequency range are well approximated by the results for the one-dimensional extensional wave in thin plates. In the present study, perturbation analysis is performed under the thin-plate approximation, and the behavior of the second-harmonic generation for the S0 mode incidence is discussed. When the imperfect joint is subjected to the incidence of a monochromatic extensional wave at angular frequency $\omega_{0}$, the resulting displacement field is written as

$$
\begin{aligned}
& u_{1}\left(x_{1}, t\right)=A_{0 \mathrm{E}} \sin \left\{\frac{\omega_{0}}{c_{1}}\left(x_{1}-c_{1} t\right)\right\}+f_{\mathrm{ref}}\left(x_{1}+c_{1} t\right), \quad x_{1}<0, \\
& u_{1}\left(x_{1}, t\right)=f_{\mathrm{tra}}\left(x_{1}-c_{1} t\right), x_{1}>0,
\end{aligned}
$$

where $A_{0 \mathrm{E}}$ is the incident amplitude, $c_{1}=2 c_{\mathrm{T}} \sqrt{1-\left(c_{\mathrm{T}} / c_{\mathrm{L}}\right)^{2}}$ is the extensional-wave velocity, and $f_{\text {ref }}\left(x_{1}+c_{1} t\right)$ and $f_{\text {tra }}\left(x_{1}-c_{1} t\right)$ represent the reflected and transmitted waves at the joint, respectively. Substitution of Eq. (17) into the boundary condition of Eq. (8) leads to a nonlinear differential equation with respect to the interfacial displacement gap $\left[u_{1}\right]$

$$
\frac{\mathrm{d}\left[u_{1}\right]}{\mathrm{d} t}+\frac{2 K_{\mathrm{N}}}{\rho c_{1}}\left[u_{1}\right]-\frac{2 \beta K_{\mathrm{N}}}{\rho c_{1}}\left[u_{1}\right]^{2}=2 \omega_{0} A_{0 \mathrm{E}} \cos \left(\omega_{0} t\right) .
$$

In a similar manner to [9], the solution of Eq. (18) is expressed as the sum of two components $\left[u_{1}\right]=\left[u_{1}^{0}\right]+\left[u_{1}^{1}\right]$. The first term $\left[u_{1}^{0}\right]$ satisfies the linearized equation

$$
\frac{\mathrm{d}\left[u_{1}^{0}\right]}{\mathrm{d} t}+\frac{2 K_{\mathrm{N}}}{\rho c_{1}}\left[u_{1}^{0}\right]=2 \omega_{0} A_{0 \mathrm{E}} \cos \left(\omega_{0} t\right),
$$

and the latter $\left[u_{1}{ }^{1}\right]$ is the perturbation which satisfies

$$
\frac{\mathrm{d}\left[u_{1}^{1}\right]}{\mathrm{d} t}+\frac{2 K_{\mathrm{N}}}{\rho c_{1}}\left[u_{1}^{1}\right]-\frac{2 \beta K_{\mathrm{N}}}{\rho c_{1}}\left[u_{1}^{0}\right]^{2}=0,
$$

when assuming sufficiently weak nonlinearity $\beta A_{0 \mathrm{E}}<<1$. By solving Eq. (19), the interfacial displacement gap by the fundamental wave component $\left[u_{1}{ }^{0}\right]$ is obtained, and 
the amplitudes of the reflected and transmitted fundamental waves can be extracted. The amplitude reflection and transmission coefficients of the fundamental extensional wave are expressed as

$$
R=\frac{1}{\sqrt{1+4 \widetilde{K}^{2}}}, \quad T=\frac{2 \widetilde{K}}{\sqrt{1+4 \widetilde{K}^{2}}},
$$

respectively, where $\widetilde{K}=K_{\mathrm{N}} /\left(\rho c_{1} \omega_{0}\right)$ is the non-dimensional stiffness of the imperfect joint. The reflection and transmission coefficients of Eq. (21) correspond to the formulae obtained by the linear analysis in the previous paper [36]. The amplitude of the transmitted second-harmonic extensional wave $A_{1 \mathrm{E}}$ can be extracted by solving Eq. (20), and its normalized amplitude $\eta_{\mathrm{E}}$ is given by

$$
\eta_{\mathrm{E}}=\frac{A_{1 \mathrm{E}}}{\beta A_{0 \mathrm{E}}^{2}}=\frac{\widetilde{K}}{\left(1+4 \widetilde{K}^{2}\right) \sqrt{1+\widetilde{K}^{2}}} .
$$

The amplitude of the reflected second-harmonic extensional wave can be obtained in a similar manner.

\section{Time-Domain Analysis}

\subsection{Modeling of Imperfect Interface}

To discuss the solutions of the perturbation analysis, the two-dimensional Navier equation of Eq. (1) is directly solved in the time domain with the nonlinear boundary conditions of Eqs. (2) and (3). Based on the interfacial stiffness of contacting surfaces measured by Drinkwater et al. [3], a power-law fitting result in [9] is used to model the dependence of the normal stiffness $K_{\mathrm{N}}$ on the nominal contact pressure. The interfacial normal stress component of Eq. (2) is given by

$$
\sigma_{11}\left(0^{ \pm}, x_{2}, t\right)=g_{\mathrm{N}}(h)=p_{0}-p(h),
$$

where $p_{0}$ is the nominal contact pressure at the equilibrium state $h=h_{0}$. The fluctuation of the contact pressure depending on the gap distance $h$ is modeled by

$$
\begin{aligned}
& p(h)=\left\{p_{0}^{1-m}-(1-m) C\left(h-h_{0}\right)\right\}^{\frac{1}{1-m}}, \quad h<h_{0}+\frac{p_{0}^{1-m}}{(1-m) C}, \\
& p(h)=0, \quad h>h_{0}+\frac{p_{0}^{1-m}}{(1-m) C},
\end{aligned}
$$

where $C$ and $m(\neq 1)$ are fitting parameters determined from the normal stiffness. In this study, the fitting parameters are set as $m=0.5$ and $C=0.06 \mathrm{MPa}^{0.5} / \mathrm{nm}$ based on [9], and the static contact pressure is fixed as $p_{0}=0.8 \mathrm{MPa}$. The relation of the nominal contact pressure $p$ to the interfacial displacement gap $h-h_{0}\left(=\left[u_{1}\right]\right)$ is shown in Fig. 2. The time-domain numerical simulation is performed with the boundary condition of Eq. JAM-19-1334 Mori et al. 
(23).

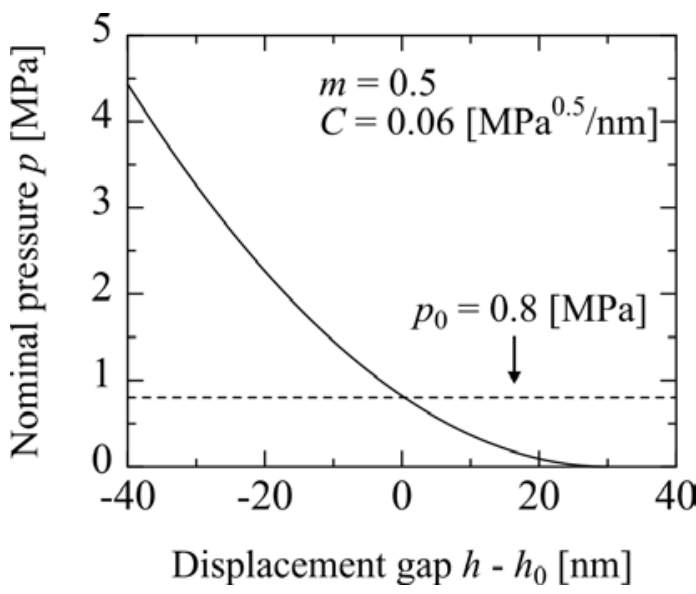

Fig. 2 Model curve of the nominal contact pressure $p$ depending on the interfacial displacement gap $h-h_{0}$

When the normal stress component is given by Eq. (23), the normal stiffness $K_{\mathrm{N}}$ and the interfacial quadratic coefficient $\beta=K_{\mathrm{NN}} / K_{\mathrm{N}}$ around the equilibrium state $h=h_{0}$ are obtained by Eq. (6) as

$$
K_{\mathrm{N}}=-\frac{\mathrm{d} p}{\mathrm{~d} h}\left(h_{0}\right)=C p_{0}^{m},
$$

and

$$
\beta=\frac{1}{2 K_{\mathrm{N}}} \frac{\mathrm{d}^{2} p}{\mathrm{~d} h^{2}}\left(h_{0}\right)=\frac{1}{2} m C p_{0}^{m-1},
$$

respectively. At $p_{0}=0.8 \mathrm{MPa}$, the value of the normalized normal stiffness is equal to $K_{\mathrm{N}} d / \mu=2.0$, where $\mu=\rho c_{\mathrm{T}}{ }^{2}$ is the shear modulus of the plates. The tangential stress component at the interface is simply assumed to be a linear function of the tangential displacement $s$ with the tangential stiffness $K_{\mathrm{T}}$, which corresponds to Eq. (9). The tangential stiffness is fixed as a constant value $K_{\mathrm{T}}=0.3 K_{\mathrm{N}}$.

\subsection{Numerical Method and Model}

A time-domain numerical simulation is carried out by the elastodynamic finite integration technique (EFIT) [41]. The schematic of the numerical model used in the time-domain analysis is shown in Fig. 3. The lengths of the two isotropic linear elastic plates are equally set as $l=400 d$, and the plates constitute an imperfect joint at $x_{1}=0$. The plate regions were discretized by square-shaped cells with side length $\Delta x=d / 20$. The total number of the cells was $20 \times 16000$. A time increment $\Delta t$ was set as $\Delta t=$ 
$\Delta x /\left(2 c_{\mathrm{L}}\right)$ in order to satisfy the stability criterion [41].

The velocity profile of the S0 mode is prescribed along the edge of the left plate $x_{1}=$ $-l$ to excite the incident S0 mode. The in-plane displacements $u_{1}$ of the incident wave and the transmitted wave across the imperfect joint $x_{1}=0$ are calculated at the locations $\mathrm{A}\left(x_{1}=-l / 2\right)$ and $\mathrm{B}\left(x_{1}=1 / 8\right)$ on the middle plane of the plates, respectively. Two types of waveforms, i.e. sinusoidal wave and pulse wave are used as input waveforms in the numerical simulations, and their results are shown separately in Sections 5.2.1 and 5.2.2.

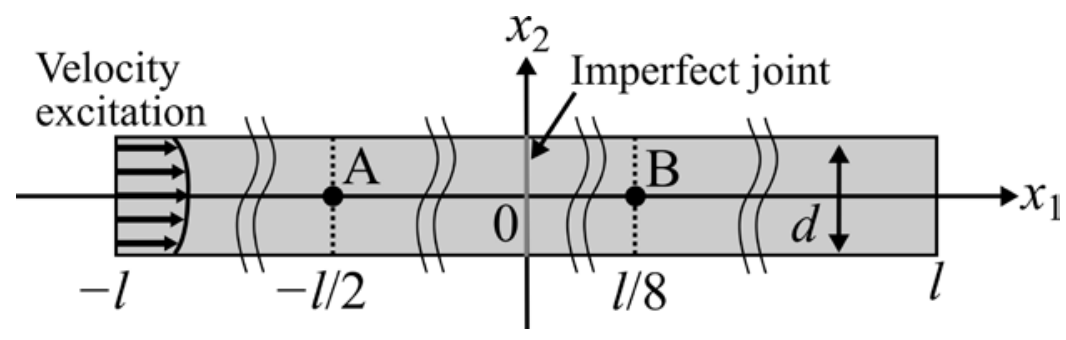

Fig. 3 Numerical model of imperfectly jointed plates in the time-domain analysis

\section{Results and Discussions}

\subsection{Perturbation Analysis}

For the S0 mode incidence, the interfacial displacement gap by the fundamental wave $\left[U_{1}{ }^{\mathrm{F}}\right]$ is obtained from Eqs. (10)-(12) by the HFEM for several normalized normal stiffnesses $K_{\mathrm{N}} d / \mu$. As reported in [36], the reflection and transmission characteristics at a linear spring-type interface for the S0 mode incidence are nominally independent of the tangential stiffness below the cut-off frequencies of the higher-order Lamb modes. Based on the interfacial displacement gap $\left[U_{1}{ }^{\mathrm{F}}\right]$, the in-plane displacement amplitude of the second-harmonic S0 mode transmitted across the joint, $A_{1}$, is calculated on the middle plane of the plate $x_{2}=0$ from Eqs. (14)-(16). Since the displacement gap $\left[U_{1}{ }^{\mathrm{F}}\right]$ is proportional to the incident amplitude $A_{0}$, the amplitudes of the second harmonics obtained from Eqs. (14)-(16) are proportional to the square of the incident amplitude. Namely, the normalized amplitude of the transmitted second-harmonic S0 mode $\eta=$ $A_{1} /\left(\beta A_{0}^{2}\right)$ does not depend on the magnitude of the incident amplitude in the perturbation analysis. The obtained second-harmonic amplitudes $\eta$ are shown for three normalized normal stiffnesses $K_{N} d / \mu$ in Fig. 4. In this figure, the horizontal axis represents the inverse of $\widetilde{K}$, in accordance with the formula in the thin-plate approximation, Eq. (22). The normalized second-harmonic amplitude $\eta$ varies gradually when $1 / \widetilde{K}$ is low, and then shows a sharp peak. The value of $1 / \widetilde{K}$ at which the second-harmonic amplitude takes a peak is found to depend on the normalized normal 
stiffness $K_{\mathrm{N}} d / \mu$. It is noted that the increase of $1 / \widetilde{K}$ corresponds to the increase of the incident frequency when the normal stiffness is fixed.

The normalized amplitude of the transmitted second-harmonic extensional wave $\eta_{\mathrm{E}}$ is calculated by Eq. (22), and is compared to the results of the second-harmonic S0 mode for different normal stiffnesses in Fig. 4. When $1 / \widetilde{K}$ is sufficiently low, the second-harmonic amplitudes of the S0 mode $\eta$ for different normal stiffnesses show good agreement with the result of the extensional wave $\eta_{\mathrm{E}}$. Namely, the normalized amplitude of the second-harmonic S0 mode $\eta$ in the low frequency range is determined simply by the non-dimensional quantity $\widetilde{K}$. This feature is analogous to the result for the second-harmonic generation by the normal incidence of the longitudinal wave shown in [9]. In other words, the low-frequency S0 mode shows nonlinear behavior similar to the longitudinal wave. However, it is found that the second-harmonic amplitude of the extensional wave $\eta_{\mathrm{E}}$ does not reproduce the peak behavior of the second-harmonic S0 mode amplitude $\eta$. This deviation results from the two-dimensional effect of the S0 mode.

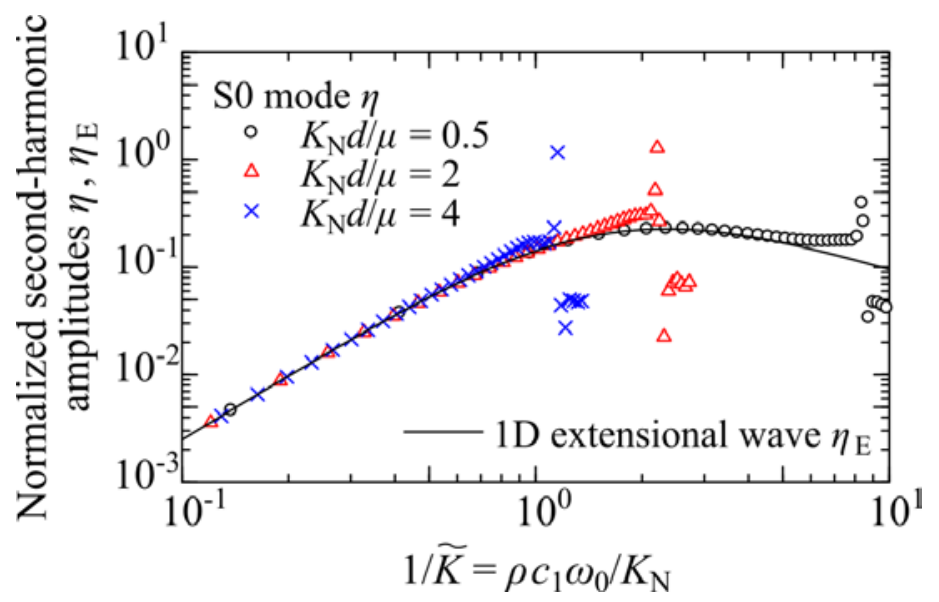

Fig. 4 Comparison of the normalized amplitude of the transmitted second-harmonic S0 mode $\eta$ obtained by the perturbation analysis using the HFEM to the result of the one-dimensional extensional wave $\eta_{\mathrm{E}}$

The amplitude of the transmitted second-harmonic S0 mode is obtained for different joint conditions in the frequency range of Eq. (7), and the incident frequency at which the second-harmonic amplitude takes a peak is extracted. At a fixed stiffness ratio $K_{\mathrm{T}} / K_{\mathrm{N}}$ $=0.3$, the relation between the normalized normal stiffness and the peak frequency is given in Table I. The peak frequencies are given as normalized quantities $f_{0} d / c_{\mathrm{T}}$. The previous paper [40] reported that the amplification of the second-harmonic S0 mode occurs due to the resonance of the imperfect joint. The resonance frequency of the 
imperfect joint is obtained by the linear analysis using the HFEM [37], and its half value is shown together in Table I. The peak frequencies shown in Table I are found to correspond to the half resonance frequency of the joint. It was shown in [37] that the imperfect joint of plates has two resonance frequencies for the S0 mode incidence, which nominally show selective dependence on the normal and tangential stiffnesses, respectively. However, at $K_{\mathrm{T}} / K_{\mathrm{N}}=0.3$, the two resonance frequencies are degenerated as a single frequency in the numerical results of the linear analysis. When the incident S0 mode of frequency $f_{0}$ interacts with the imperfect joint, the vibration of the double frequency $2 f_{0}$ is induced at the joint surfaces due to the nonlinear effect according to Eq. (15). If the frequency $2 f_{0}$ corresponds to the resonance frequency of the imperfect joint $f_{\mathrm{r}}$, i.e. $2 f_{0}=f_{\mathrm{r}}$, the amplification of the second-harmonic S0 mode occurs.

Table I Relation of the normalized normal stiffness $K_{N} d / \mu$ to the normalized peak frequency of the second-harmonic S0 mode amplitude $\eta$ at a fixed stiffness ratio $K_{\mathrm{T}} / K_{\mathrm{N}}=0.3$. Half resonance frequencies obtained by the linear analysis using the HFEM are shown together for comparison.

\begin{tabular}{ccc}
\hline$K_{N} d / \mu$ & Peak frequency & Half resonance frequency \\
\hline 0.05 & 0.38 & 0.38 \\
0.5 & 0.39 & 0.39 \\
2 & 0.41 & 0.41 \\
4 & 0.42 & 0.42 \\
\hline
\end{tabular}

At a fixed normal stiffness $K_{N} d / \mu=2$, the relation of the peak frequency to the stiffness ratio $K_{\mathrm{T}} / K_{\mathrm{N}}$ is shown in Table II, together with the half resonance frequencies of the imperfect joint. The peak frequencies correspond to the half of the resonance frequency which is nominally invariant with the tangential stiffness, as shown in Table II. This feature seems to appear due to the driving term $\beta K_{\mathrm{N}} Y_{1}^{2} \exp \left\{-\mathrm{i}\left(2 \phi_{1}\right)\right\} / 2$ in the interfacial normal stress component of Eq. (15). The amplification by the resonance in the tangential direction does not occur because no excitation term emerges at the double frequency in the tangential stress component of Eq. (16).

JAM-19-1334 Mori et al. 
Table II Relation of the stiffness ratio $K_{\mathrm{T}} / K_{\mathrm{N}}$ to the normalized peak frequency of the second harmonic S0 mode amplitude $\eta$ at a fixed normal stiffness $K_{N} d / \mu=2$. Half resonance frequencies obtained by the linear analysis using the HFEM are shown together for comparison.

\begin{tabular}{ccc}
\hline$K_{\mathrm{T}} / K_{\mathrm{N}}$ & Peak frequency & Half resonance frequencies \\
\hline 0.2 & 0.41 & $0.39,0.41$ \\
0.3 & 0.41 & 0.41 \\
0.6 & 0.41 & $0.41,0.42$ \\
\hline
\end{tabular}

\subsection{Time-Domain Analysis}

\subsubsection{Sinusoidal Wave Incidence}

To discuss the perturbation analysis results in Section 5.1, the nonlinear behavior for the sinusoidal wave incidence is analyzed by the time-domain numerical simulation. Since the EFIT is formulated by using particle velocities $v_{\alpha}\left(x_{1}, x_{2}, t\right)$, the S0 mode is excited by prescribing the particle velocities at the left edge of the plate $v_{\alpha}\left(-l, x_{2}, t\right)$ as

$$
v_{\alpha}\left(-l, x_{2}, t\right)=\frac{\partial u_{\alpha}^{0}\left(x_{2}, t\right)}{\partial t}
$$

in both normal and tangential directions $(\alpha=1,2)$, where

$$
\begin{aligned}
& u_{\alpha}^{0}\left(x_{2}, t\right)=a_{0} \exp \left\{-\left(\frac{t-t_{\mathrm{c}}}{\sigma_{\mathrm{c}}}\right)^{2}\right\} \sin \left(2 \pi f_{0} t\right) U_{\alpha}^{\mathrm{S} 0}\left(x_{2}, f_{0}\right), \quad 0<t<t_{\mathrm{c}}, \\
& u_{\alpha}^{0}\left(x_{2}, t\right)=a_{0} \sin \left(2 \pi f_{0} t\right) U_{\alpha}^{\mathrm{S} 0}\left(x_{2}, f_{0}\right), \quad t \geq t_{\mathrm{c}},
\end{aligned}
$$

is the displacement component, $t_{\mathrm{c}}=3 \sigma_{\mathrm{c}}=6 \mu \mathrm{s}$, and $U_{\alpha}^{\mathrm{S} 0}\left(x_{2}, f_{0}\right)$ is the $x_{\alpha}$-direction displacement profile of the S0 mode at the frequency $f_{0}$. At a steady state $t \geq t_{\mathrm{c}}$, the above displacement is expressed as the sinusoidal function of the amplitude $\mid a_{0} U_{\alpha}{ }^{\mathrm{S} 0}\left(x_{2}\right.$, $\left.f_{0}\right) \mid$ with respect to time. The coefficient $a_{0}$ is determined if the displacement amplitude of the incident wave $A_{0}$ is given.

The in-plane displacements $u_{1}$ of the incident wave and the transmitted wave across the imperfect joint are obtained at the locations $A$ and $B$, respectively. At $f_{0} d / c_{\mathrm{T}}=0.4$, (i.e. $f_{0}=1.28 \mathrm{MHz}$ ), the waveforms calculated at the locations $\mathrm{A}$ and $\mathrm{B}$ are shown in Fig. 5(a) and (b), respectively, for the incident amplitude $A_{0}=3 \mathrm{~nm}$. The incident amplitude $A_{0}$ is obtained as the in-plane displacement amplitude of the incident wave calculated at the location A in a steady state, in accordance with the perturbation analysis using the HFEM. The horizontal axis represents the normalized time $t c_{T} / d$, and the vertical axis the normalized displacement $u_{1} / A_{0}$. After reaching the steady state, each waveform is 
multiplied by a Hanning window of the normalized time length 95, and its amplitude spectrum is obtained by fast Fourier transform (FFT). The amplitude spectra of the incident and transmission waveforms are shown in Fig. 5(c). The vertical axis is normalized by the maximum amplitude of the incident wave. The amplitude spectrum of the incident wave has a peak around at $f d / c_{\mathrm{T}}=0.4$, while the transmitted wave shows three other clear peaks, which correspond to the DC component, the second harmonic at $f d / c_{\mathrm{T}}=0.8$, and the third harmonic around at $f d / c_{\mathrm{T}}=1.2$. These components are generated due to the nonlinear effect of the imperfect joint. For the input frequency $f_{0} d / c_{\mathrm{T}}=0.4$, the amplitudes of the transmitted fundamental S0 mode $A_{0 \mathrm{~T}}$ and the generated second-harmonic S0 mode $A_{1}$ are obtained as the amplitude spectrum values of the transmission waveform at $f d / c_{\mathrm{T}}=0.4$ and $f d / c_{\mathrm{T}}=0.8$, respectively. 
(a)

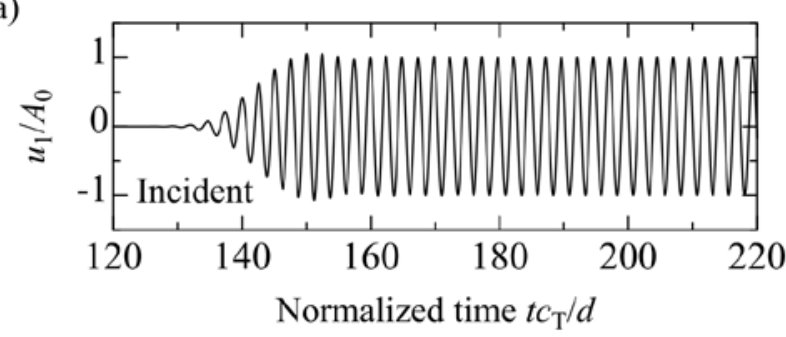

(b)
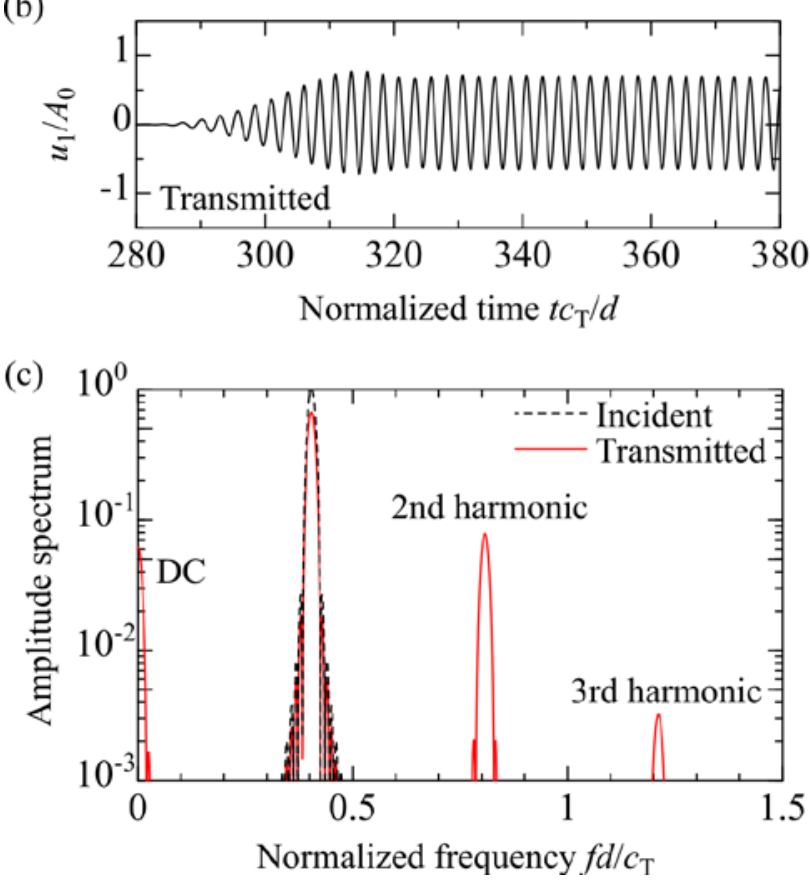

Fig. 5 In-plane displacements of (a) the incident wave and (b) the transmitted wave across the imperfect joint at the input frequency $f_{0} d / c_{\mathrm{T}}=0.4$ for the incident amplitude $A_{0}=3 \mathrm{~nm}$, calculated by the EFIT at the two locations A and B, respectively. The amplitude spectra of the incident and transmitted waves at steady states are shown in (c) as functions of the normalized frequency.

The above numerical calculation is run for different input frequencies in $f_{0} d / c_{\mathrm{T}}<0.5$ at the incident amplitude $A_{0}=3 \mathrm{~nm}$. The transmission coefficient of the fundamental wave $T=A_{0 \mathrm{~T}} / A_{0}$ and the normalized second-harmonic amplitude $\eta=A_{1} /\left(\beta A_{0}^{2}\right)$ are calculated for each input frequency and are shown in Fig. 6(a) and (b), respectively. The results of the S0 mode and the extensional wave obtained by the perturbation analyses are shown together in these figures. In Fig. 6(a), the transmission coefficient of the S0 mode obtained by the perturbation analysis decreases monotonically with the input frequency and is shown to be in good agreement with the result by the EFIT on the whole. This behavior is fairly reproduced by the transmission coefficient of the extensional wave, which is obtained by Eq. (21). In Fig. 6(b), the normalized amplitudes of the second-harmonic S0 mode calculated by the perturbation analysis and the EFIT 
are in good agreement. These agreements imply that the calculation parameters in the EFIT satisfy the assumption of weak nonlinearity in the perturbation analysis. The perturbation analysis result of the second-harmonic extensional wave cannot reproduce the amplification behavior of the S0 mode in Fig. 6(b), which has been shown in Fig. 4.

(a)

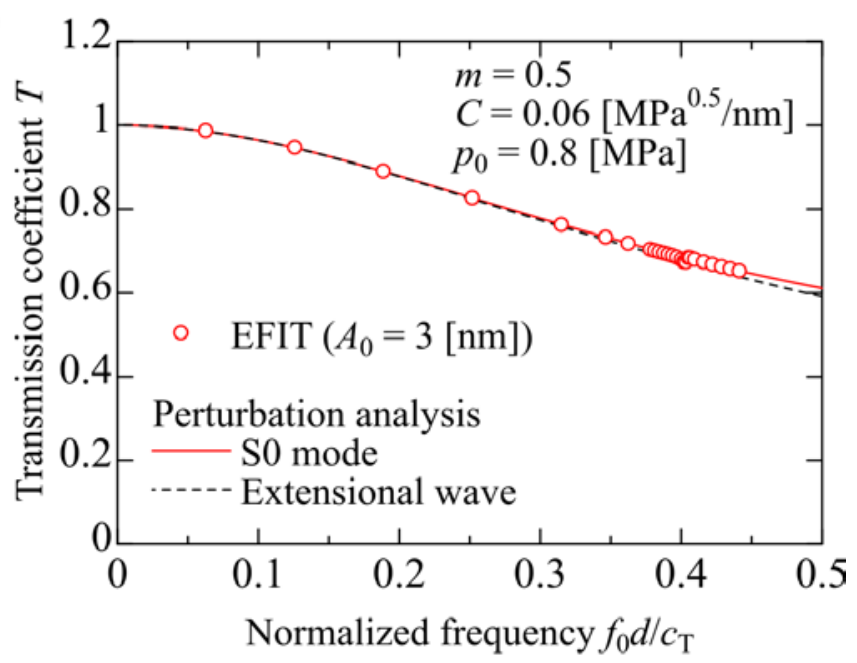

(b)

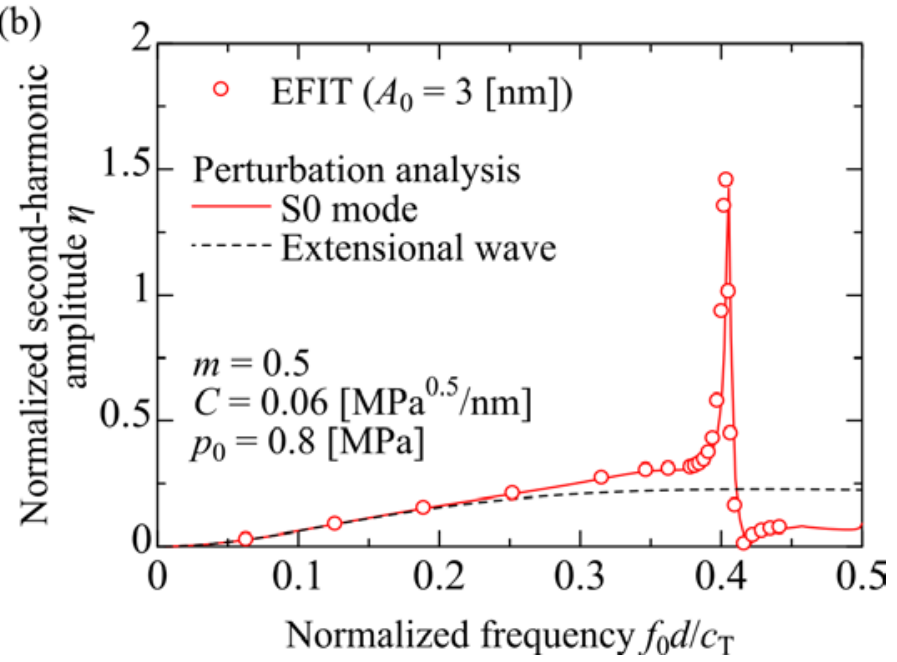

Fig. 6 Variation of (a) the transmission coefficient of the fundamental wave $T$ and (b) the normalized second-harmonic amplitude $\eta$ obtained by the EFIT with the input frequency for the incident amplitude $A_{0}=3 \mathrm{~nm}$, together with the perturbation analysis results of the $\mathrm{S} 0$ mode and the extensional wave

To investigate the effect of the incident amplitude $A_{0}$, the numerical simulation is carried out for relatively large amplitudes $A_{0}=12 \mathrm{~nm}$ and $A_{0}=18 \mathrm{~nm}$. The transmission coefficients $T$ and normalized second-harmonic amplitudes $\eta$ obtained by the time-domain analysis are shown in Fig. 7(a) and (b), respectively. The increase of the incident amplitude enhances the nonlinear effect and leads to deviation from the results 
for weak nonlinearity in both figures. In Fig. 7(a), the curves of the transmission coefficient for the incident amplitudes $A_{0}=12 \mathrm{~nm}$ and $A_{0}=18 \mathrm{~nm}$ are located below the one for $A_{0}=3 \mathrm{~nm}$. It is inferred that for large incident amplitudes, a non-negligible amount of energy is transferred from the fundamental wave to the higher-order harmonics due to the nonlinear effect. This transition could bring about the decrease of the transmission coefficient of the fundamental wave from the value for weak nonlinearity.

(a)

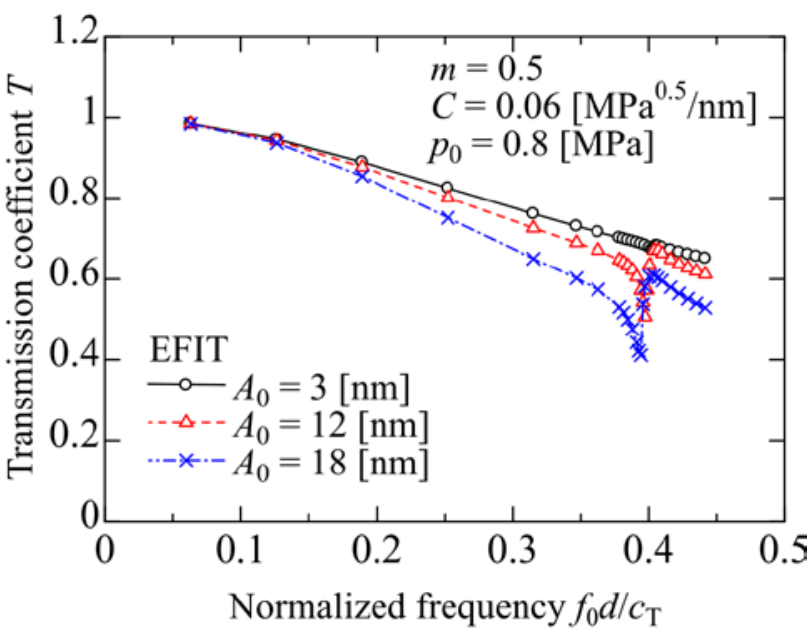

(b)

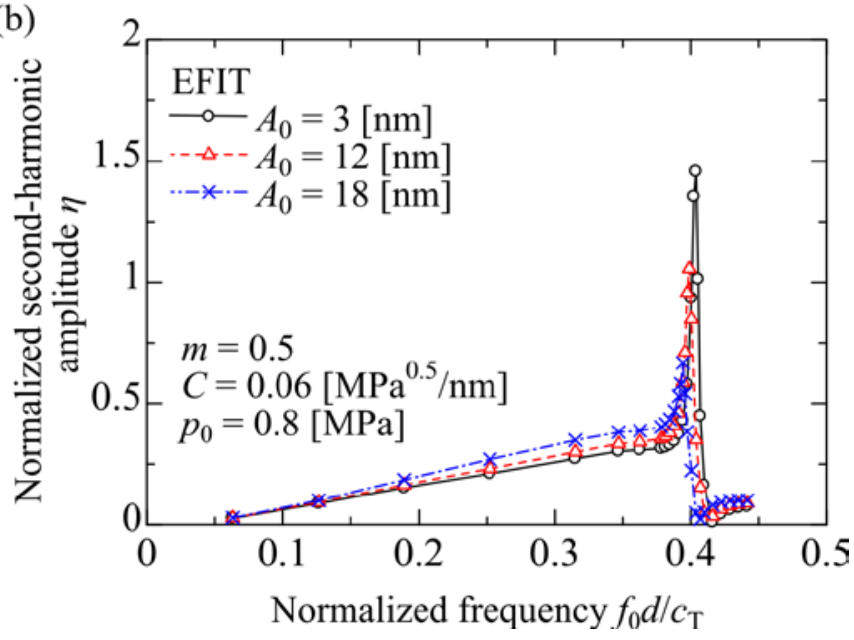

Fig. 7 Variation of (a) the transmission coefficient of the fundamental wave $T$ and (b) the normalized second-harmonic amplitude $\eta$ with the incident frequency for different incident amplitudes $A_{0}$, obtained by the EFIT

The transmission coefficients for the incident amplitudes $A_{0}=12 \mathrm{~nm}$ and $A_{0}=18$ $\mathrm{nm}$ in Fig. 7 (a) clearly show valleys at around $f_{0} d / c_{\mathrm{T}}=0.4$, which are slightly seen in the result for $A_{0}=3 \mathrm{~nm}$ in Fig. 6(a) as well. It is likely that this phenomenon occurs due to the amplification of the second harmonic. In Fig. 7(b), the normalized second-harmonic 
amplitude $\eta$ shows a peak at around $f_{0} d / c_{\mathrm{T}}=0.4$ even if the incident amplitude $A_{0}$ is increased. The peak frequency shifts slightly to the left as the incident amplitude is increased. The decrease of the resonance frequency by the increase of the excitation amplitude can be confirmed in various nonlinear vibration systems [46-49], and Fig. 7(b) shows that the similar phenomenon appears in the resonance of the imperfect joint by the S0 mode incidence. The peak frequencies of the second-harmonic amplitudes for $A_{0}=12 \mathrm{~nm}$ and $A_{0}=18 \mathrm{~nm}$ are found to correspond to the valley frequencies of the transmission coefficients in Fig. 7(a). The local minimum of the transmission coefficient could appear because more power is transferred from the fundamental wave to the higher-order harmonics at the peak frequency than at the other input frequencies.

At the peak frequency of the second-harmonic amplitude for each incident amplitude in Fig. 7(b), the time variation of the interfacial normal stress $\sigma_{11}$ on the middle plane of the plate at a steady state is shown in Fig. 8(a). The stress components are normalized by the nominal contact pressure value at the equilibrium state, $p_{0}$. From Eq. (23) and Fig. 2, the normal stress $\sigma_{11}$ does not exceed $p_{0}$, i.e. $\sigma_{11} \leq p_{0}$. In Fig. 8(a), the interfacial stress is lower than $p_{0}$ when $A_{0}=3 \mathrm{~nm}$ and $A_{0}=12 \mathrm{~nm}$. However, the interfacial stress for $A_{0}=18 \mathrm{~nm}$ reaches $p_{0}$ and its peaks are flattened. Namely, the nonlinear effect is more significant at the incident amplitude $A_{0}=18 \mathrm{~nm}$. 
(a)

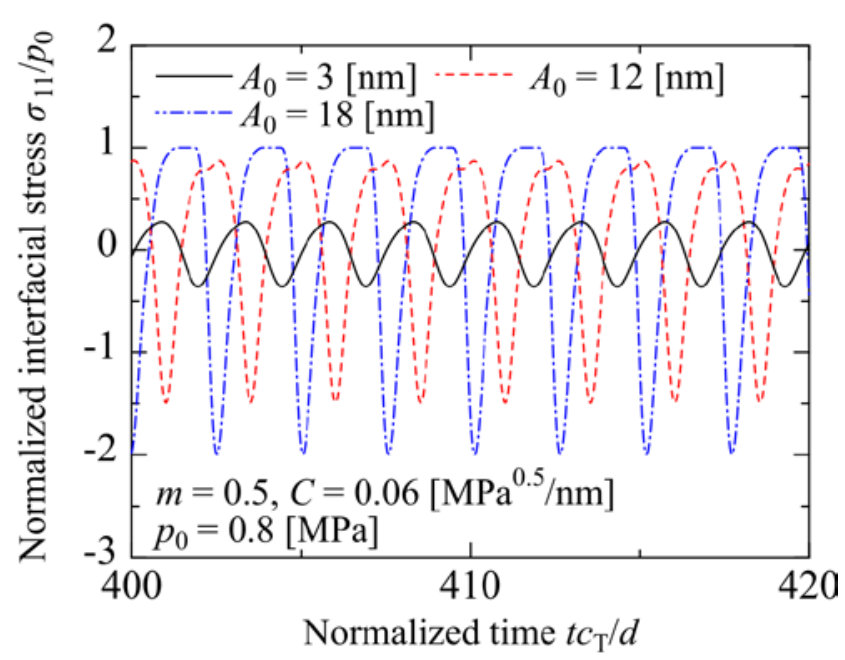

(b)

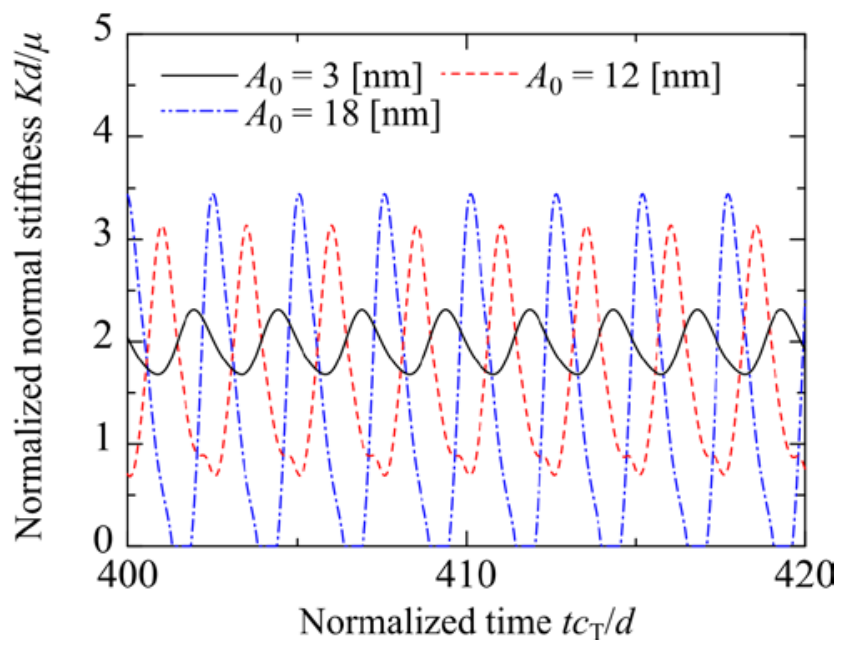

Fig. 8 Time histories of (a) the normalized interfacial stress $\sigma_{11} / p_{0}$ on the middle plane of the plate and (b) the normalized normal stiffness $K d / \mu$ at steady states for different incident amplitudes $A_{0}$, when the input frequencies $f_{0}$ correspond to the peak frequencies of the second-harmonic amplitude shown in Fig. 7(b), respectively

The shift of the peak frequency by the increase of the incident amplitude in Fig. 7(b) is discussed based on the equivalent normal stiffness of the imperfect joint. A time-dependent quantity

$$
K(t)=\frac{\mathrm{d} \sigma_{11}}{\mathrm{~d} h}=-\frac{\mathrm{d} p}{\mathrm{~d} h},
$$

is calculated for each incident amplitude from the interfacial normal stress on the middle plane of the plate in Fig. 8(a). At the equilibrium state $h=h_{0}, K(t)$ corresponds to $K_{\mathrm{N}}$ in Eq. (25). The time variations of the normal stiffness $K(t)$ at the steady states are shown as normalized quantities in Fig. 8(b) for different incident amplitudes. The JAM-19-1334 Mori et al. 
time-averaged normal stiffness

$$
K_{\text {ave }}=\frac{1}{T} \int_{t_{0}}^{t_{0}+T} K(t) \mathrm{d} t
$$

is calculated for each incident amplitude, where the time integration is numerically carried out in the time range of Fig. 8(b), i.e. $t_{0}=400 d / c_{\mathrm{T}}$ and $T=20 \mathrm{~d} / \mathrm{c}_{\mathrm{T}}$. For each time-averaged normal stiffness $K_{\text {ave }}$, the resonance frequency of the imperfect joint is calculated by the linear analysis using the HFEM. The half value of the obtained resonance frequency is shown as the predicted peak frequency in Fig. 9, together with the actual results by the EFIT. The peak frequency obtained by the EFIT shows decreasing behavior with increasing incident amplitude, which is reproduced in a qualitative manner by the results predicted from $K_{\text {ave }}$. The increase of the incident amplitude results in the decrease of the equivalent normal stiffness, which leads to the decrease of the predicted peak frequency. If the sensitivity of the second-harmonic amplitude to the variation of the normal stiffness is taken into account, the shift of the peak frequency in Fig. 9 could be discussed quantitatively. However, this topic is not further explored in the present study.

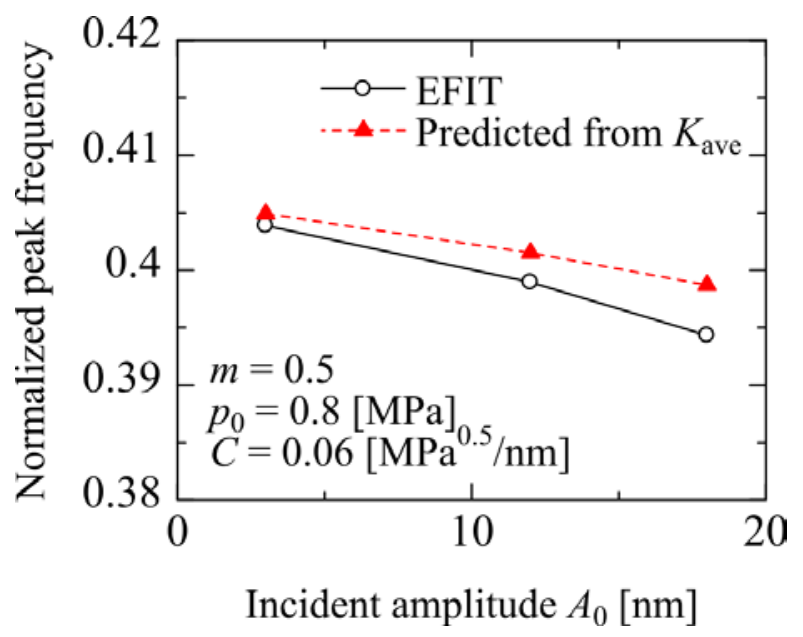

Fig. 9 Relation of the peak frequencies of the second-harmonic amplitude calculated by the EFIT to the incident amplitude, together with the results predicted by the time-averaged normal stiffness $K_{\text {ave }}$

\subsubsection{Pulse Wave Incidence}

In this section, the transient behavior of Lamb waves interacting with the nonlinear joint is examined. The S0 mode is excited by prescribing the particle velocities at the left edge of the plate according to Eq. (27). The displacement components $u_{\alpha}^{0}\left(x_{2}, t\right)$ are given by a Gaussian-modulated tone-burst signal with the center frequency $f_{0}$ 


$$
u_{\alpha}^{0}\left(x_{2}, t\right)=a_{0} \exp \left\{-\left(\frac{t-t_{\mathrm{c}}}{\sigma_{\mathrm{c}}}\right)^{2}\right\} \sin \left[2 \pi f_{0}\left(t-t_{\mathrm{c}}\right)\right] U_{\alpha}^{\mathrm{So}}\left(x_{2}, f_{0}\right),
$$

where the parameters $t_{\mathrm{c}}$ and $\sigma_{\mathrm{c}}$ are the same as those in Eq. (28). The numerical simulation is performed for three different center frequencies, $f_{0} d / c_{T}=0.30,0.40$, and 0.45. The coefficient $a_{0}$ is determined for each center frequency in order that the maximum in-plane displacement $U_{1}$ of the incident wave calculated at the location $\mathrm{A}$ is $U_{1}=6 \mathrm{~nm}$.

For the pulse wave incidence at $f_{0} d / c_{\mathrm{T}}=0.30$, the in-plane displacement $u_{1}$ of the transmitted wave calculated at the location B is shown in Fig. 10(a). The displacement is normalized by the maximum displacement of the incident wave $U_{1}$. The calculated waveform is analyzed by short-time fast Fourier transform (STFT) using a Hanning window of normalized time length 32 to obtain the time-frequency relation of the amplitude spectrum. The calculated amplitude spectrum is shown in Fig. 10(b). The horizontal and vertical axes represent the normalized time and the normalized frequency, respectively. The maximum amplitude of the fundamental wave is set as $0 \mathrm{~dB}$ in Fig. 10(b), and the data which are lower than $-50 \mathrm{~dB}$ are not shown in the figure. As a result, not only the fundamental wave of frequency $f d / c_{\mathrm{T}}=0.3$ but also the second harmonic at $\mathrm{fd} / \mathrm{c}_{\mathrm{T}}=0.6$ and the DC component are found to appear in the transmission waveform. The peak value of the second harmonic in Fig. 10(b) is approximately $-40 \mathrm{~dB}$. 
(a)

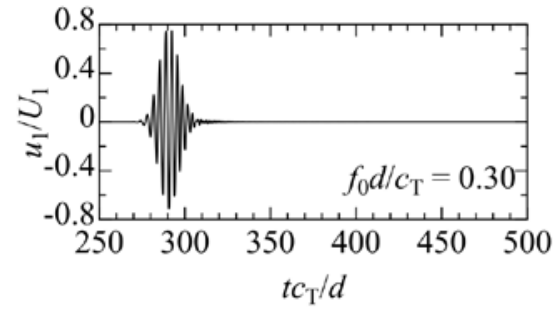

(b)

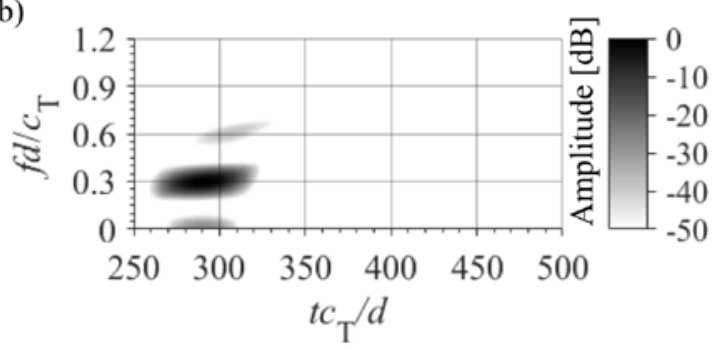

(c)

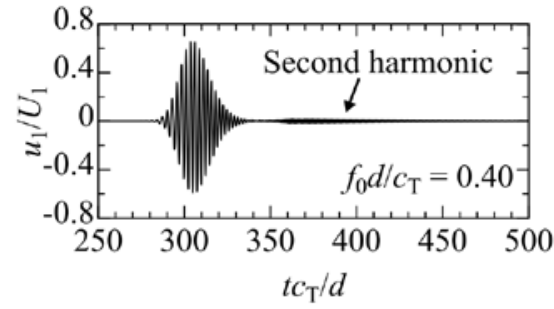

(d)

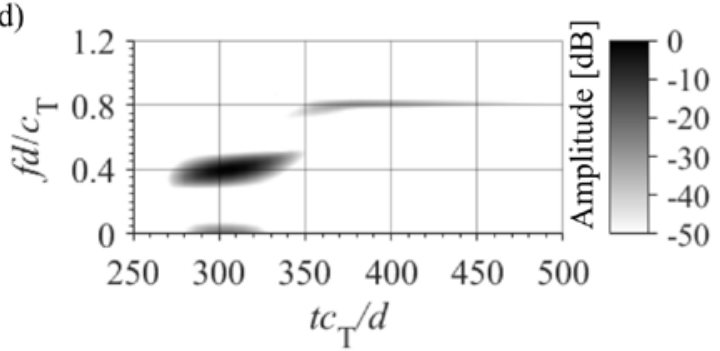

(e)

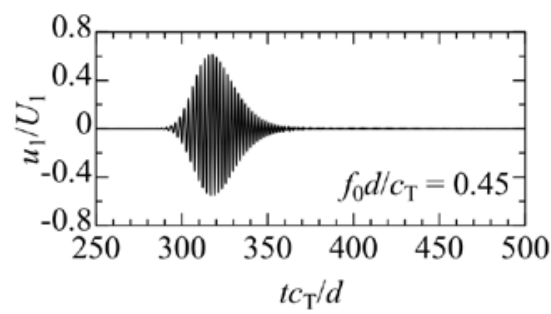

(f)

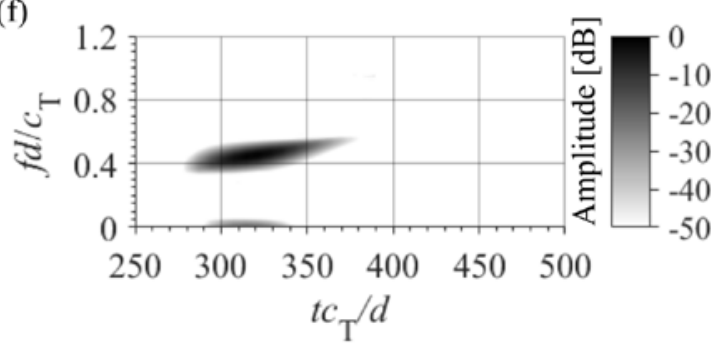

Fig. 10 In-plane displacements and their time-frequency relations of the transmitted waves for the pulse wave incidence at three different center frequencies: (a) and (b) $f_{0} d / c_{\mathrm{T}}=0.30$, (c) and (d) $f_{0} d / c_{\mathrm{T}}=0.40$, and (e) and (f) $f_{0} d / c_{\mathrm{T}}=0.45$. The in-plane displacements are shown in (a), (c), and (e), and the time-frequency relations are in (b), (d), and (f).

Likewise, the above numerical simulation is carried out for the other two center frequencies. The transmission waveform and its STFT result for the center frequency $f_{0} d / c_{\mathrm{T}}=0.40$ are shown in Fig. 10(c) and (d), respectively, and those for $f_{0} d / c_{\mathrm{T}}=0.45$ are shown in Fig. 10(e) and (f), respectively. The amplitude spectra in Fig. 10(d) and (f) are normalized by the maximum value in Fig. 10(b). For the center frequency of $f_{0} d / c_{\mathrm{T}}=$ 0.40 , the amplitude spectrum shows the second-harmonic component after $t c_{\mathrm{T}} / d=350$ in Fig. 10(d), which can be confirmed in the transmission waveform of Fig. 10(c). The second-harmonic component shows a long-time oscillation, and its amplitude spectrum value in Fig. 10(d) is approximately $-30 \mathrm{~dB}$. This value is roughly $10 \mathrm{~dB}$ higher than 
the amplitude spectrum of the second harmonic for $f_{0} d / c_{T}=0.30$ in Fig. 10 (b). On the other hand, when the center frequency is $f_{0} d / c_{T}=0.45$, the amplitude spectrum of the second harmonic becomes lower than $-50 \mathrm{~dB}$ in Fig. 10(f).

The second-harmonic generation for the pulse wave incidence is associated with the second-harmonic amplitude for the sinusoidal wave incidence which has been shown in Fig. 7(b). If the sinusoidal wave is incident and its amplitude is sufficiently low, the incident frequency at which the second-harmonic amplitude takes a peak corresponds to the half resonance frequency of the imperfect joint, as discussed in Section 5.1. At $p_{0}=$ $0.8 \mathrm{MPa}$, the resonance frequency of the joint is obtained as $f d / c_{\mathrm{T}}=0.81$ by the linear analysis using the HFEM. For the pulse wave incidence, among the three center frequencies, $f_{0} d / c_{T}=0.40$ is the closest to the half resonance frequency. Therefore the second harmonic for $f_{0} d / c_{\mathrm{T}}=0.40$ is expected to show the highest amplitude among the three cases due to the effect of the resonance, which corresponds to the amplitude spectra in Fig. 10(b), (d), and (f). The previous paper [50] reported that for the S0 mode incidence around at the resonance frequency, the transmission waveform at the linear imperfect joint shows a long oscillation tail. The long oscillation tail of the second-harmonic component for $f_{0} d / c_{\mathrm{T}}=0.40$ in Fig. 10(c) and (d) implies the occurrence of the joint resonance by the nonlinear effect.

\section{Concluding Remarks}

In this study, the harmonic generation of Lamb waves at a nonlinear imperfect joint of plates has been investigated by perturbation analysis and time-domain numerical simulation. The imperfect joint has been modeled as a nonlinear spring-type interface, which yields the interfacial stress components as functions of the displacement discontinuities. For sufficiently weak nonlinearity, the generation behavior of the second harmonic has been analyzed by perturbation analysis. For the incidence of the lowest-order symmetric (S0) mode, the perturbation analysis results have shown that the amplitude of the transmitted second-harmonic s0 mode can be reproduced by the thin-plate approximation using the one-dimensional extensional wave in a low frequency range. However, in a relatively high frequency range, the second-harmonic amplitude of the S0 mode has been shown to take a sharp peak, which is not reproduced by the thin-plate approximation. This phenomenon has been found to occur when the frequency of the second harmonic corresponds to the resonance frequency of the imperfect joint. Furthermore, the time-domain numerical simulation using the elastodynamic finite integration technique (EFIT) has been carried out to discuss the results by the perturbation analysis. For the sinusoidal wave incidence, if the amplitude 
of the incident wave is sufficiently low, the results by the time-domain analysis have been in good agreement with the perturbation analysis results of the S0 mode. However, as the incident amplitude is increased, the time-domain numerical results have deviated from the results for weak nonlinearity and the peak frequency of the second-harmonic amplitude has decreased. For the pulse wave incidence, it has been found that the waveform of the transmitted second-harmonic S0 mode shows a long oscillation tail when it is amplified due to the joint resonance.

\section{Acknowledgments}

This work has been supported by Grant-in-Aids for Scientific Research (KAKENHI) Grant Nos. JP18K13663 and JP19H02026 from Japan Society for the Promotion of Science (JSPS).

\section{References}

[1] Kendall, K., and Tabor, D., 1971, “An Ultrasonic Study of the Area of Contact between Stationary and Sliding Surfaces,” Proc. R. Soc. A Math. Phys. Eng. Sci., 323(1554), pp. 321-340.

[2] Nagy, P. B., 1992, “Ultrasonic Classification of Imperfect Interfaces,” J. Nondestruct. Eval., 11(3-4), pp. 127-139.

[3] Drinkwater, B. W., Dwyer-Joyce, R. S., and Cawley, P., 1996, “A Study of the Interaction between Ultrasound and a Partially Contacting Solid-Solid Interface,” Proc. R. Soc. A Math. Phys. Eng. Sci., 452(1955), pp. 2613-2628.

[4] Brotherhood, C. J., Drinkwater, B. W., and Guild, F. J., 2002, “The Effect of Compressive Loading on the Ultrasonic Detectability of Kissing Bonds in Adhesive Joints,” J. Nondestruct. Eval., 21(3), pp. 95-104.

[5] Richardson, J. M., 1979, "Harmonic Generation at an Unbonded Interface-I. Planar Interface between Semi-Infinite Elastic Media,” Int. J. Eng. Sci., 17(1), pp. 73-85.

[6] Solodov, I. Y., 1998, "Ultrasonics of Non-Linear Contacts: Propagation, Reflection and NDE-Applications,” Ultrasonics, 36, pp. 383-390.

[7] Donskoy, D., Sutin, A., and Ekimov, A., 2001, "Nonlinear Acoustic Interaction on Contact Interfaces and Its Use for Nondestructive Testing,” NDT E Int., 34(4), pp. 231-238.

[8] Pecorari, C., 2003, "Nonlinear Interaction of Plane Ultrasonic Waves with an Interface between Rough Surfaces in Contact,” J. Acoust. Soc. Am., 113(6), p. 3065-3072.

[9] Biwa, S., Nakajima, S., and Ohno, N., 2004, “On the Acoustic Nonlinearity of 
Solid-Solid Contact with Pressure-Dependent Interface Stiffness,” J. Appl. Mech., 71, pp. 508-515.

[10] Ohara, Y., Mihara, T., and Yamanaka, K., 2006, "Effect of Adhesion Force between Crack Planes on Subharmonic and DC Responses in Nonlinear Ultrasound,” Ultrasonics, 44(2), pp. 194-199.

[11] Biwa, S., Hiraiwa, S., and Matsumoto, E., 2006, "Experimental and Theoretical Study of Harmonic Generation at Contacting Interface," Ultrasonics, 44, pp. e1319-e1322.

[12] Kim, J.-Y., and Lee, J.-S., 2007, “A Micromechanical Model for Nonlinear Acoustic Properties of Interfaces between Solids,” J. Appl. Phys., 101(4), 043501.

[13] Nam, T., Lee, T., Kim, C., Jhang, K.-Y., and Kim, N., 2012, “Harmonic Generation of an Obliquely Incident Ultrasonic Wave in Solid-Solid Contact Interfaces,” Ultrasonics, 52(6), pp. 778-783.

[14] Blanloeuil, P., Meziane, A., and Bacon, C., 2014, "Numerical Study of Nonlinear Interaction between a Crack and Elastic Waves under an Oblique Incidence,” Wave Motion, 51(3), pp. 425-437.

[15] Yan, D., Drinkwater, B. W., and Neild, S. A., 2009, "Measurement of the Ultrasonic Nonlinearity of Kissing Bonds in Adhesive Joints,” NDT E Int., 42(5), pp. 459-466.

[16] Shui, G., Wang, Y., Huang, P., and Qu, J., 2015, "Nonlinear Ultrasonic Evaluation of the Fatigue Damage of Adhesive Joints,” NDT E Int., 70, pp. 9-15.

[17] Ju, T., Achenbach, J. D., Jacobs, L. J., and Qu, J., 2019, “One-Way Mixing of Collinear Waves in an Adhesive Layer,” J. Acoust. Soc. Am., 145(1), pp. 110-120.

[18] Biwa, S., and Ishii, Y., 2016, "Second-Harmonic Generation in an Infinite Layered Structure with Nonlinear Spring-Type Interfaces,” Wave Motion, 63, pp. $55-67$.

[19] Ishii, Y., Biwa, S., and Adachi, T., 2018, "Second-Harmonic Generation in a Multilayered Structure with Nonlinear Spring-Type Interfaces Embedded between Two Semi-Infinite Media,” Wave Motion, 76, pp. 28-41.

[20] de Lima, W. J. N., and Hamilton, M. F., 2003, "Finite-Amplitude Waves in Isotropic Elastic Plates,” J. Sound Vib., 265(4), pp. 819-839.

[21] Deng, M., 2003, “Analysis of Second-Harmonic Generation of Lamb Modes Using a Modal Analysis Approach,” J. Appl. Phys., 94(6), pp. 4152-4159.

[22] Müller, M. F., Kim, J.-Y., Qu, J., and Jacobs, L. J., 2010, “Characteristics of Second Harmonic Generation of Lamb Waves in Nonlinear Elastic Plates,” J. Acoust. Soc. Am., 127(4), pp. 2141-2152.

[23] Matsuda, N., and Biwa, S., 2011, "Phase and Group Velocity Matching for JAM-19-1334 Mori et al. 
Cumulative Harmonic Generation in Lamb Waves,” J. Appl. Phys., 109(9), 94903.

[24] Hong, M., Su, Z., Wang, Q., Cheng, L., and Qing, X., 2014, “Modeling Nonlinearities of Ultrasonic Waves for Fatigue Damage Characterization: Theory, Simulation, and Experimental Validation,” Ultrasonics, 54(3), pp. 770-778.

[25] Shkerdin, G., and Glorieux, C., 2010, “Nonlinear Clapping Modulation of Lamb Modes by Normally Closed Delamination,” IEEE Trans. Ultrason. Ferroelectr. Freq. Control, 57(6), pp. 1426-1433.

[26] Shen, Y., and Giurgiutiu, V., 2013, "Predictive Modeling of Nonlinear Wave Propagation for Structural Health Monitoring with Piezoelectric Wafer Active Sensors,” J. Intell. Mater. Syst. Struct., 25(4), pp. 506-520.

[27] Hong, M., Su, Z., Lu, Y., Sohn, H., and Qing, X., 2015, "Locating Fatigue Damage Using Temporal Signal Features of Nonlinear Lamb Waves,” Mech. Syst. Signal Process., 60, pp. 182-197.

[28] Ciampa, F., Scarselli, G., Pickering, S., and Meo, M., 2015, “Nonlinear Elastic Wave Tomography for the Imaging of Corrosion Damage," Ultrasonics, 62, pp. 147-155.

[29] Delrue, S., and Van Den Abeele, K., 2015, "Detection of Defect Parameters Using Nonlinear Air-Coupled Emission by Ultrasonic Guided Waves at Contact Acoustic Nonlinearities,” Ultrasonics, 63, pp. 147-154.

[30] Yelve, N. P., Mitra, M., and Mujumdar, P. M., 2015, “Detection of Stiffener Disbonding in a Stiffened Aluminium Panel Using Nonlinear Lamb Wave,” Appl. Acoust., 89, pp. 267-272.

[31] Shen, Y., and Cesnik, C. E. S., 2017, "Modeling of Nonlinear Interactions between Guided Waves and Fatigue Cracks Using Local Interaction Simulation Approach,” Ultrasonics, 74, pp. 106-123.

[32] Zhou, J., Xiao, L., Qu, W., and Lu, Y., 2017, "Nonlinear Lamb Wave Based DORT Method for Detection of Fatigue Cracks,” NDT E Int., 92, pp. 22-29.

[33] Yang, Y., Ng, C.-T., Kotousov, A., Sohn, H., and Lim, H. J., 2018, “Second Harmonic Generation at Fatigue Cracks by Low-Frequency Lamb Waves: Experimental and Numerical Studies,” Mech. Syst. Signal Process., 99, pp. 760-773.

[34] Biwa, S., Kishiwada, S., Inserra, C., and Matsumoto, E., 2010, "Modeling of Flexural Wave Propagation in a Plate with Contacting Interfaces,” J. Solid Mech. Mater. Eng., 4(8), pp. 1186-1197.

[35] Biwa, S., 2019, "Second-harmonic generation at contacting interfaces," Nonlinear Ultrasonic and Vibro-Acoustical Techniques for Nondestructive Evaluation, Springer, New York, pp. 263-299. 
[36] Mori, N., Biwa, S., and Hayashi, T., 2013, "Reflection and Transmission of Lamb Waves at an Imperfect Joint of Plates,” J. Appl. Phys., 113(7), 074901.

[37] Mori, N., and Biwa, S., 2015, "Resonance of an Imperfect Joint of Plates by the Lowest-Order Symmetric Lamb Mode,” J. Acoust. Soc. Am., 137(6), pp. 3139-3148.

[38] Mori, N., and Biwa, S., 2016, "Transmission of Lamb Waves and Resonance at an Adhesive Butt Joint of Plates,” Ultrasonics, 72, pp. 80-88.

[39] Mori, N., and Biwa, S., 2017, "Transmission Characteristics of the S0 and A0 Lamb Waves at Contacting Edges of Plates,” Ultrasonics, 81, pp. 93-99.

[40] Mori, N., Biwa, S., and Kusaka, T., 2018, "Numerical Study of the Second Harmonic Generation of Lamb Waves at an Imperfect Joint of Plates,” Proc. Meeting Acoust., 34, 030002.

[41] Fellinger, P., Marklein, R., Langenberg, K. J., and Klaholz, S., 1995, "Numerical

Modeling of Elastic Wave Propagation and Scattering with EFIT-Elastodynamic Finite Integration Technique,” Wave motion, 21, pp. 47-66.

[42] An, Z., Wang, X., Deng, M., Mao, J., and Li, M., 2013, “A Nonlinear Spring Model for an Interface between Two Solids,” Wave Motion, 50(2), pp. 295-309.

[43] Yoshioka, N., and Scholz, C. H., 1989, "Elastic Properties of Contacting Surfaces under Normal and Shear Loads: 1. Theory,” J. Geophys. Res., 94(B12), pp. 17681-17690.

[44] Królikowski, J., and Szczepek, J., 1993, “Assessment of Tangential and Normal Stiffness of Contact between Rough Surfaces Using Ultrasonic Method,” Wear, 160(2), pp. 253-258.

[45] Biwa, S., Hiraiwa, S., and Matsumoto, E., 2007, "Stiffness Evaluation of Contacting Surfaces by Bulk and Interface Waves,” Ultrasonics, 47, pp. 123-129.

[46] Landau, L. D., and Lifshitz, E. M., 1960, Mechanics, Pergamon Press, New York.

[47] Johnson, P. A., Zinszner, B., and Rasolofosaon, P. N. J., 1996, "Resonance and elastic nonlinear phenomena in rock,” J. Geophys. Res., 101(B5), pp. 11553-11564.

[48] Chen, J., Kim, J.-Y., Kurtis, K. E., and Jacobs, L. J., 2011, “Theoretical and Experimental Study of the Nonlinear Resonance Vibration of Cementitious Materials with an Application to Damage Characterization,” J. Acoust. Soc. Am., 130(5), pp. 2728-2737.

[49] Solodov, I., 2014, "Resonant Acoustic Nonlinearity of Defects for Highly-Efficient Nonlinear NDE,” J. Nondestruct. Eval., 33(2), pp. 252-262.

[50] Mori, N., and Biwa, S., 2015, "Interaction of Lamb Waves with an Imperfect Joint of Plates: Reflection, Transmission and Resonance,” Phys. Procedia, 70(7), pp. 480-483. 


\section{Figure Captions}

Fig. 1 Nonlinear imperfect joint of isotropic linear elastic plates at $x_{1}=0$

Fig. 2 Model curve of the nominal contact pressure $p$ depending on the interfacial displacement gap $h-h_{0}$

Fig. 3 Numerical model of imperfectly jointed plates in the time-domain analysis

Fig. 4 Comparison of the normalized amplitude of the transmitted second-harmonic S0 mode $\eta$ obtained by the perturbation analysis using the HFEM to the result of the one-dimensional extensional wave $\eta_{\mathrm{E}}$

Fig. 5 In-plane displacements of (a) the incident wave and (b) the transmitted wave across the imperfect joint at the input frequency $f_{0} d / c_{T}=0.4$ for the incident amplitude $A_{0}=3 \mathrm{~nm}$, calculated by the EFIT at the two locations A and B, respectively. The amplitude spectra of the incident and transmitted waves at steady states are shown in (c) as functions of the normalized frequency.

Fig. 6 Variation of (a) the transmission coefficient of the fundamental wave $T$ and (b) the normalized second-harmonic amplitude $\eta$ obtained by the EFIT with the input frequency for the incident amplitude $A_{0}=3 \mathrm{~nm}$, together with the perturbation analysis results of the S0 mode and the extensional wave

Fig. 7 Variation of (a) the transmission coefficient of the fundamental wave $T$ and (b) the normalized second-harmonic amplitude $\eta$ with the incident frequency for different incident amplitudes $A_{0}$, obtained by the EFIT

Fig. 8 Time histories of (a) the normalized interfacial stress $\sigma_{11} / p_{0}$ on the middle plane of the plate and (b) the normalized normal stiffness $K d / \mu$ at steady states for different incident amplitudes $A_{0}$, when the input frequencies $f_{0}$ correspond to the peak frequencies of the second-harmonic amplitude shown in Fig. 7(b), respectively

Fig. 9 Relation of the peak frequencies of the second-harmonic amplitude calculated by the EFIT to the incident amplitude, together with the results predicted by the time-averaged normal stiffness $K_{\text {ave }}$

Fig. 10 In-plane displacements and their time-frequency relations of the transmitted waves for the pulse wave incidence at three different center frequencies: (a) and (b) $f_{0} d / c_{\mathrm{T}}=0.30$, (c) and (d) $f_{0} d / c_{\mathrm{T}}=0.40$, and (e) and (f) $f_{0} d / c_{\mathrm{T}}=0.45$. The in-plane displacements are shown in (a), (c), and (e), and the time-frequency relations are in (b), (d), and (f). 


\section{Table Captions}

Table I Relation of the normalized normal stiffness $K_{N} d / \mu$ to the normalized peak frequency of the second-harmonic S0 mode amplitude $\eta$ at a fixed stiffness ratio $K_{\mathrm{T}} / K_{\mathrm{N}}=0.3$. Half resonance frequencies obtained by the linear analysis using the HFEM are shown together for comparison.

Table II Relation of the stiffness ratio $K_{\mathrm{T}} / K_{\mathrm{N}}$ to the normalized peak frequency of the second harmonic S0 mode amplitude $\eta$ at a fixed normal stiffness $K_{N} d / \mu=2$. Half resonance frequencies obtained by the linear analysis using the HFEM are shown together for comparison. 